\title{
Generation of magnetic seed fields in self-gravitating protogalactic clouds
}

\author{
G. T. Birk ${ }^{1,2}$, H. Wiechen ${ }^{2,3}$, and H. Lesch ${ }^{1,2}$ \\ 1 Institute for Astronomy and Astrophysics, University of Munich, Germany \\ 2 Centre of Interdisciplinary Plasma Science, Garching, Germany \\ 3 Max-Planck-Institut für extraterrestrische Physik, Garching, Germany
}

Received 14 March 2002 / Accepted 11 July 2002

\begin{abstract}
The seed magnetization of protogalaxies can be provided by relative shear flows and collisional friction of the ionized and the neutral fluid components in partially ionized self-gravitating and rotating protogalactic clouds. The gravitational potential of a collapsing protogalactic cloud is the ultimate energy source that partly is converted to magnetic fields and the associated electric currents by collisional momentum transfer in the partially ionized systems. Self-consistent plasma-neutral gas simulations, that take into account self-gravitation, show that magnetic fields of the order of some $10^{-14} \mathrm{G}$ are generated on spatial scales of some $100 \mathrm{pc}$ in about $7 \times 10^{6}$ years.
\end{abstract}

Key words. magnetic fields - galaxies: formation - methods: numerical

\section{Introduction}

Large-scale magnetic fields of the order of $\mu G$ are common in galaxies and galaxy clusters (e.g. Kronberg 1994; Beck et al. 1996). It is worth mentioning that magnetic fields in very young objects like the damped Ly $\alpha$ systems observed at red-shifts of $z \approx 2-3$ and nearby spiral galaxies have similar field strengths (Kronberg et al. 1992; Wolfe et al. 1992). In other words galactic magnetic fields are obviously produced by some plasma mechanisms on time scales of a few $10^{8}$ years or may be even faster. In principle, these fields can be understood as a consequence of some dynamo process together with field amplification due to the global galactic collapse (e.g. Lesch \& Chiba 1997 and references therein). A crucial problem of galactic magnetic fields concerns the origin of the seed magnetic fields which are a necessary pre-requisite for any dynamo action to start with (Rees 1987; Lesch \& Chiba 1995). Amplification to $\mu \mathrm{G}$ strengths on time scales of the order of $10^{8} \mathrm{yr}$ demands at least $10^{-20}-10^{-15} \mathrm{G}$ seed fields depending on the dynamo mechanism (Lesch \& Chiba 1995).

Taking the standard picture that galaxies origin from the merging of numerous rotating clouds consisting of a partially ionized plasma embedded in neutral gas, a natural mechanism for the self-generation of magnetic fields in galaxies is the nonideal momentum transfer between the ionized and the neutral fluid components in relative sheared flows (Lesch et al. 1989; Huba \& Fedder 1993; Birk et al. 1996). To be more

Send offprint requests to: G. T. Birk, e-mail: birk@usm.uni-muenchen.de specific, we follow the argumentation by Haehnelt et al. (1996) or Rauch et al. (1997) that high red-shift damped Ly $\alpha$ systems are formed by numerous gas clumps in the framework of hierarchical structure formation. In a previous paper (Wiechen et al. 1998; hereafter referenced as Paper I) we presented the first step towards a self-consistent modelling of the generation of magnetic fields in evolved damped Ly $\alpha$ systems consisting of protogalactic clumps. These clumps are discussed as the building blocks in the progenitors of today spiral galaxies. There, we discussed the magnetic field generation mechanism in some detail in the context of the derivation of the relevant balance equations, in particular, the induction equation (Eq. (5) below) that governs the dynamical evolution of the magnetic field. Numerical simulations of rotating partially ionized protogalactic gases, assuming parameters typical for rotating protogalactic clumps in Ly $\alpha$ clouds, showed that, in principle, magnetic fields of the order of $10^{-15} \mathrm{G}$ can be generated on time scales of $10^{6} \mathrm{yr}$. This result was also found by including some constant external gravitational force at the centers of the rotating clumps (Wiechen et al. 1999, 2000). This first model introduced some assumptions about the relative shear flow between the ionized and neutral gas components in an ad hoc manner. The flow can be interpreted as the consequence of intrinsic angular momentum and tidal interactions as driving forces. However, the question of the energy source was only addressed incidentally in Paper I and by Wiechen et al. (1999, 2000). In the present contribution we investigate the magnetic field self-generation during the initial collapse dynamics of rotating partially ionized protogalactic clumps in a self-consistent 
way. The numerical model that includes self-gravitation explicitly is introduced in the next section. In Sect. 3 the numerical results are presented. Finally, we discuss our findings in Sect. 4.

\section{Numerical model}

In our simulations the following set of normalized plasma neutral gas equations (see also Paper I) are numerically integrated by means of an explicit finite differences code (Birk \& Otto 1996) that is supplemented by self-gravitation

$$
\begin{aligned}
& \frac{\partial \rho}{\partial t}=-\nabla \cdot(\mathbf{v} \rho) \\
& \frac{\partial \rho_{\mathrm{n}}}{\partial t}=-\nabla \cdot\left(\mathbf{v}_{\mathrm{n}} \rho_{\mathrm{n}}\right)
\end{aligned}
$$

$\frac{\partial}{\partial t}(\rho \mathbf{v})=-\nabla \cdot(\rho \mathbf{v v})-\nabla p+\nabla \times \mathbf{B} \times \mathbf{B}-\rho \nabla \Psi-\rho v_{\mathrm{pn}}\left(\mathbf{v}-\mathbf{v}_{\mathrm{n}}\right)$

$\frac{\partial}{\partial t}\left(\rho_{\mathrm{n}} \mathbf{v}_{\mathrm{n}}\right)=-\nabla \cdot\left(\rho_{\mathrm{n}} \mathbf{V}_{\mathrm{n}} \mathbf{v}_{\mathrm{n}}\right)-\nabla p_{\mathrm{n}}-\rho_{\mathrm{n}} \nabla \Psi-\rho_{\mathrm{n}} \nu_{\mathrm{np}}\left(\mathbf{v}_{\mathrm{n}}-\mathbf{v}\right)$

$$
\frac{\partial \mathbf{B}}{\partial t}=\nabla \times(\mathbf{v} \times \mathbf{B})-\nabla \times(\eta \nabla \times \mathbf{B})-\nabla \times \hat{v}_{\mathrm{en}}\left(\mathbf{v}_{\mathrm{n}}-\mathbf{v}\right)
$$

$$
\begin{aligned}
\frac{\partial p}{\partial t}= & -\mathbf{v} \cdot \nabla p-\gamma p \nabla \cdot \mathbf{v}+(\gamma-1)\left(2 \eta(\nabla \times \mathbf{B})^{2}\right. \\
& \left.-3 v_{\mathrm{pn}}\left(p-\frac{\rho}{\rho_{\mathrm{n}}} p_{\mathrm{n}}\right)+\rho v_{\mathrm{pn}}\left(\mathbf{v}-\mathbf{v}_{\mathrm{n}}\right)^{2}\right)
\end{aligned}
$$

$$
\begin{aligned}
\frac{\partial p_{\mathrm{n}}}{\partial t}= & -\mathbf{v}_{\mathrm{n}} \cdot \nabla p_{\mathrm{n}}-\gamma_{\mathrm{n}} p_{\mathrm{n}} \nabla \cdot \mathbf{v}_{\mathrm{n}}+\left(\gamma_{\mathrm{n}}-1\right) \\
& \times\left(3 v_{\mathrm{np}}\left(p_{\mathrm{n}}-\frac{\rho_{\mathrm{n}}}{\rho} p\right)+\rho_{\mathrm{n}} v_{\mathrm{np}}\left(\mathbf{v}_{\mathrm{n}}-\mathbf{v}\right)^{2}\right)
\end{aligned}
$$

$$
\Delta \Psi=G\left(\rho+\rho_{\mathrm{n}}\right),
$$

where $\rho, \mathbf{v}, p, \mathbf{B}$ and $\Psi$ denote the mass density, bulk velocity, thermal pressure, magnetic field and gravitational potential. The index " $n$ " represents the neutral gas quantities. The effective plasma-neutral gas collision frequency is $v_{\mathrm{pn}}$ where $\nu_{\mathrm{pn}} \rho=\nu_{\mathrm{np}} \rho_{\mathrm{n}}$ holds to guarantee momentum conservation. In the induction equation (Eq. (5)) $\hat{v}_{\mathrm{en}}=v_{\mathrm{en}} \mathrm{cm}_{\mathrm{e}} / c$ with the electron-neutral elastic collision frequency, normalized speed of light, electron mass and elementary charge. By $\eta, \gamma$ and $G$ the collisional resistivity, ratio of specific heats and the gravitational constant are denoted. It is the final term in the induction equation which results in the self-magnetization caused by relative sheared plasma-neutral gas flows. Normalization of the physical quantities is carried out by typical plasma mass density, a typical length scale, the plasma sound speed, and the plasma sound time scale. The magnetic pressure is normalized to the thermal one. The latter is measured by a typical number density and the kinetic temperature. Equation (8) considers the self-gravitation which yields the explicit energy source. The gravitational potential results from both the neutral and ionized components of the considered partially ionized plasma.

The numerical code makes use of a modified Leapfrog and a Dufort-Frankel integration schemes. The source terms in the

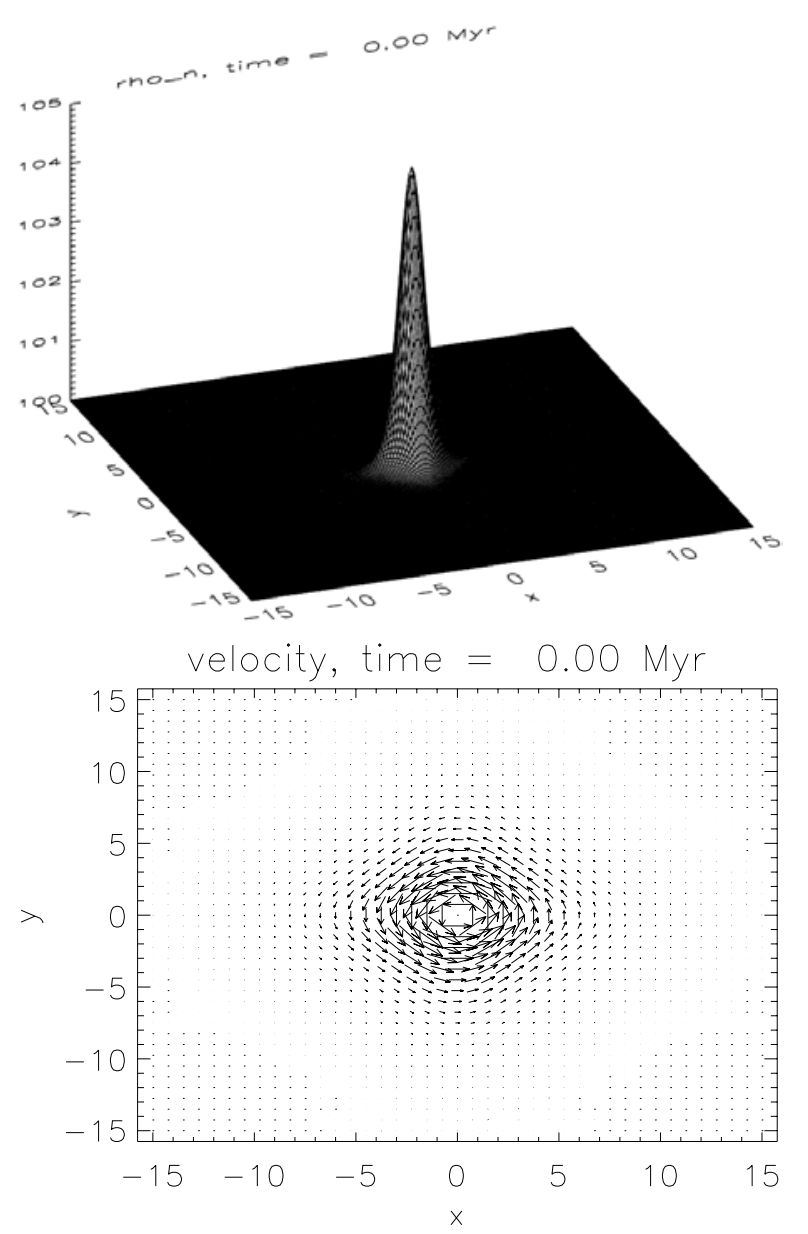

Fig. 1. The initial mass density for the neutral gas fluid component and the initial vortical flow pattern.

balance equations for the mass densities, momentums and thermal pressures are treated in some flux-corrected manner (Birk \& Otto 1996). Poisson's equation is integrated by a standard multigrid procedure.

The initial configuration of our simulation studies is characterized by the following parameters (see also Paper I): for the isothermal $\left(T=T_{\mathrm{n}}=1\right)$ homogeneously distributed mass densities we assume $\rho=1$ and $\rho_{\mathrm{n}}=100$ normalized to a mass density of $1 m_{\mathrm{p}} \mathrm{cm}^{-3}$ where $m_{\mathrm{p}}$ is the proton mass. Thermal equilibrium of the neutral and the ionized components is chosen for simplicity. Different temperatures are probably more realistic. For comparison, simulation runs have been performed for $T=10 T_{\mathrm{n}}$ and $T=40 T_{\mathrm{n}}$ (see end of Sect. 3). One should note that the Saha equation determines the ionization rate for strict thermodynamic equilibrium only. In many astrophysical plasmas, however, the rates of collisional transitions are not higher than the rates of radiative transitions, and for the relevant transitions the radiation field does not correspond to a cavity radiation of the local kinetic temperature, either. The neutral gas density is isobarically perturbed with an amplitude of $5 \%$ of the initial homogeneous density and with a $\cosh ^{-2}(x) \cosh ^{-2}(y)$-profile (Fig. 1, upper plot). Within a cold dark matter scenario one may interpret such a density perturbation as a result of some external gravitation caused by this unknown matter component. Alternatively, a density 


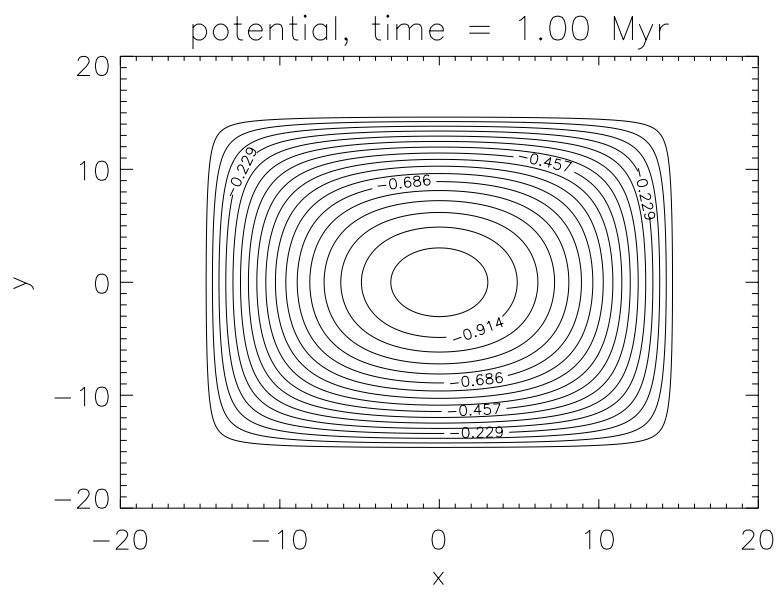

Fig. 2. Contour plot of the gravitational potential after $t=1 \mathrm{Myr}$.

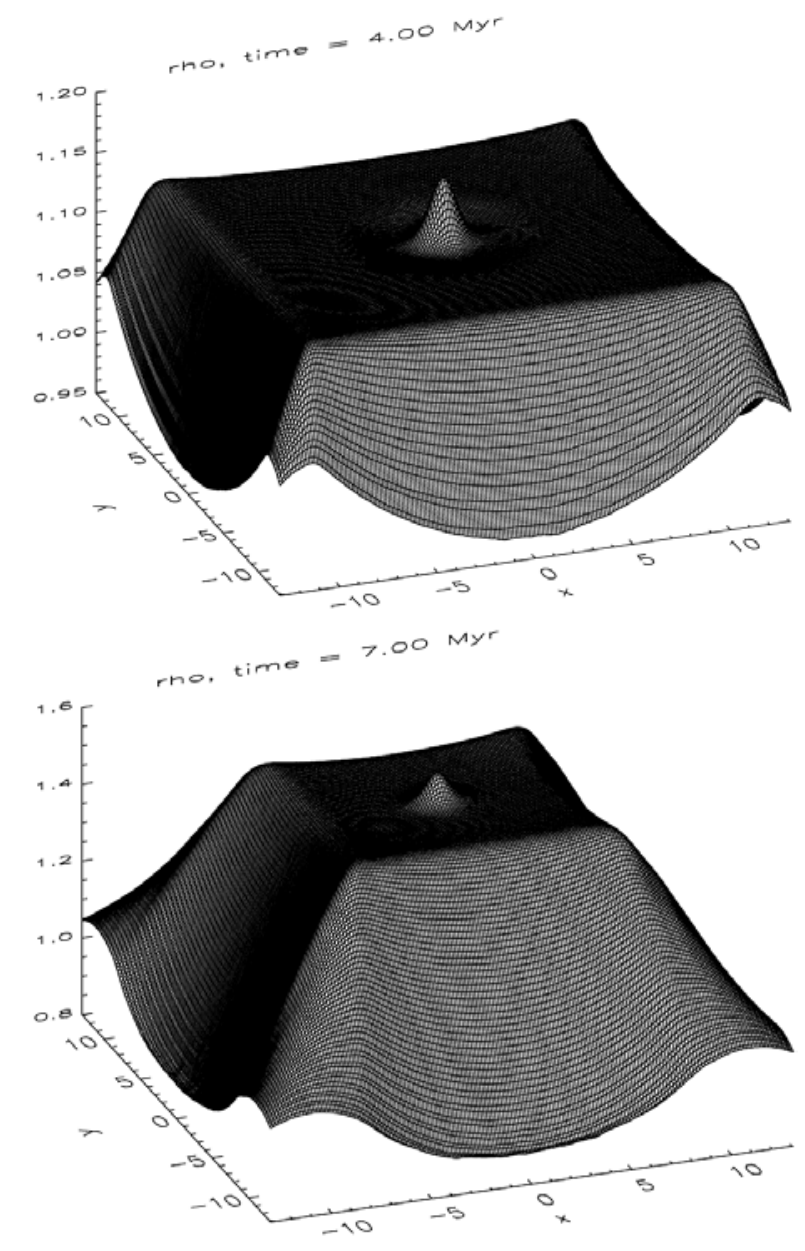

Fig. 3. Surface plot of the mass density of the ionized fluid component after $t=4 \mathrm{Myr}$ (upper plot) and $t=7 \mathrm{Myr}$ (lower plot).

perturbation could be chosen in the ionized fluid component or in both the plasma and neutral gas. Any density perturbation leads to the dynamics caused by the locally enhanced self-gravitational potential as discussed in the following. Time scales are measured by the sound time scale $t=L / c_{\mathrm{s}}$ where $L=25 \mathrm{pc}$ is the typical length scale and $c_{\mathrm{s}}=10^{6} \mathrm{~cm} \mathrm{~s}^{-1}$ is the sound speed. For the initial velocity we choose a planar vortical flow pattern (Fig. 1, lower plot) with a maximum velocity
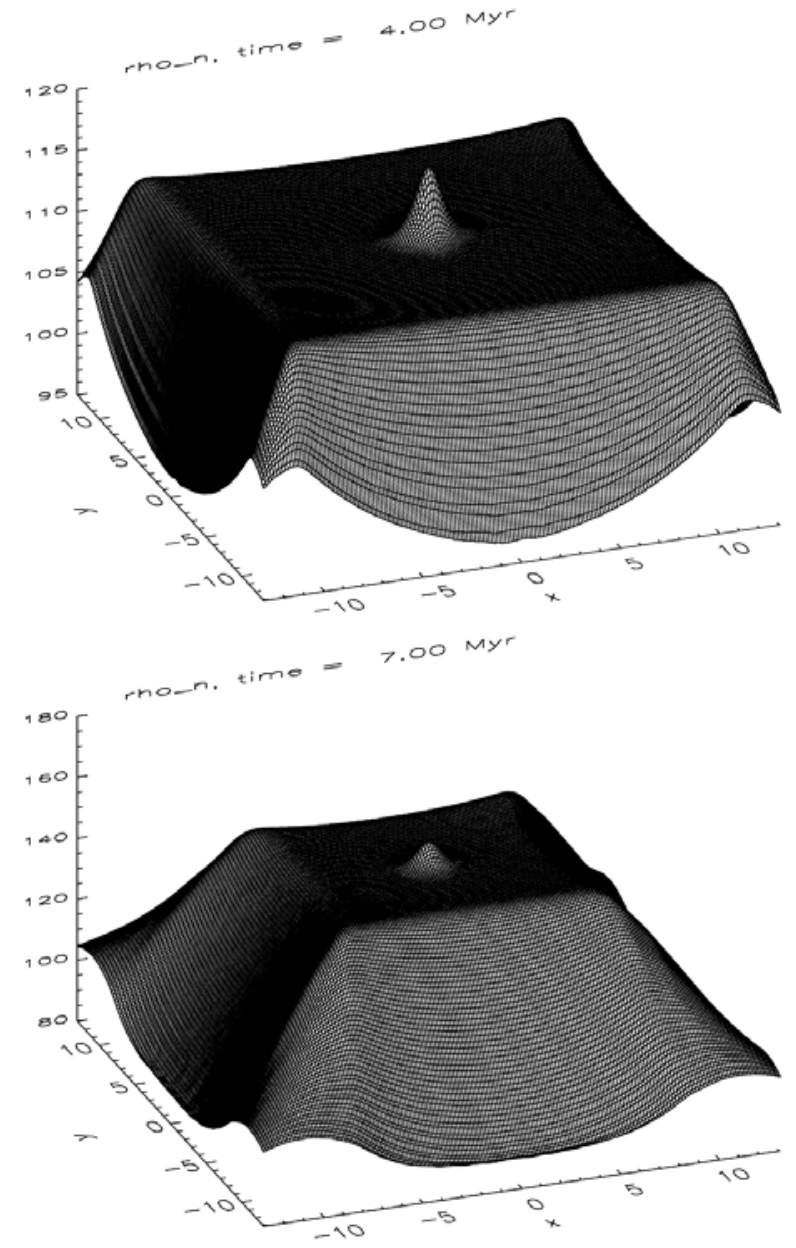

Fig. 4. Surface plot of the mass density of the neutral fluid component after $t=4 \mathrm{Myr}$ (upper plot) and $t=7 \mathrm{Myr}$ (lower plot).

corresponding to $1 \mathrm{~km} \mathrm{~s}^{-1}$. This is not an equilibrium solution, since the non-linear convective terms in the momentum balance Eqs. (3) and (4) do not vanish. However, the violation of the equilibrium results in a small force directed radially outward the vortex and is over-compensated by the gravitational pull. The initial configuration is unstable in terms of a linear mode analysis of the gravitational instability (Goldreich \& LyndenBell 1964). The collisional momentum transfer between the plasma and the neutral gas components is measured by the effective collision frequency of about $2 \times 10^{-7} \mathrm{~s}^{-1}$, if one uses the elastic collision frequency between the charged particles and neutral hydrogen (Huba 1998) $v_{(\mathrm{e}, \mathrm{i}) \mathrm{H}}=n_{\mathrm{H}} \sigma\left(k T_{(\mathrm{e}, \mathrm{i})} / m_{(\mathrm{e}, \mathrm{i})}\right)^{1 / 2}$ with a scattering cross section of $\sigma \simeq 4 \times 10^{-15} \mathrm{~cm}^{-2}$ and $k, T_{\mathrm{H}}$ denoting the Boltzmann constant and the hydrogen temperature, respectively. For numerical reasons we have to underestimate $v_{\mathrm{pn}}$ by a factor of $1 / 100$. However, several runs with different values of $v_{\mathrm{pn}}$ result in almost the same generated magnetic field strength at different stages of the collapse dynamics. We note, that the time scales do not depend linearly on the collision frequencies. A higher collision frequency results in a faster momentum transfer but also in a higher magnetic field generation rate. The self-gravitational potential causes a continuous acceleration. Consequently, due to the different pressure forces (see Figs. 3 and 4) for the plasma and the neutral gas fluids 

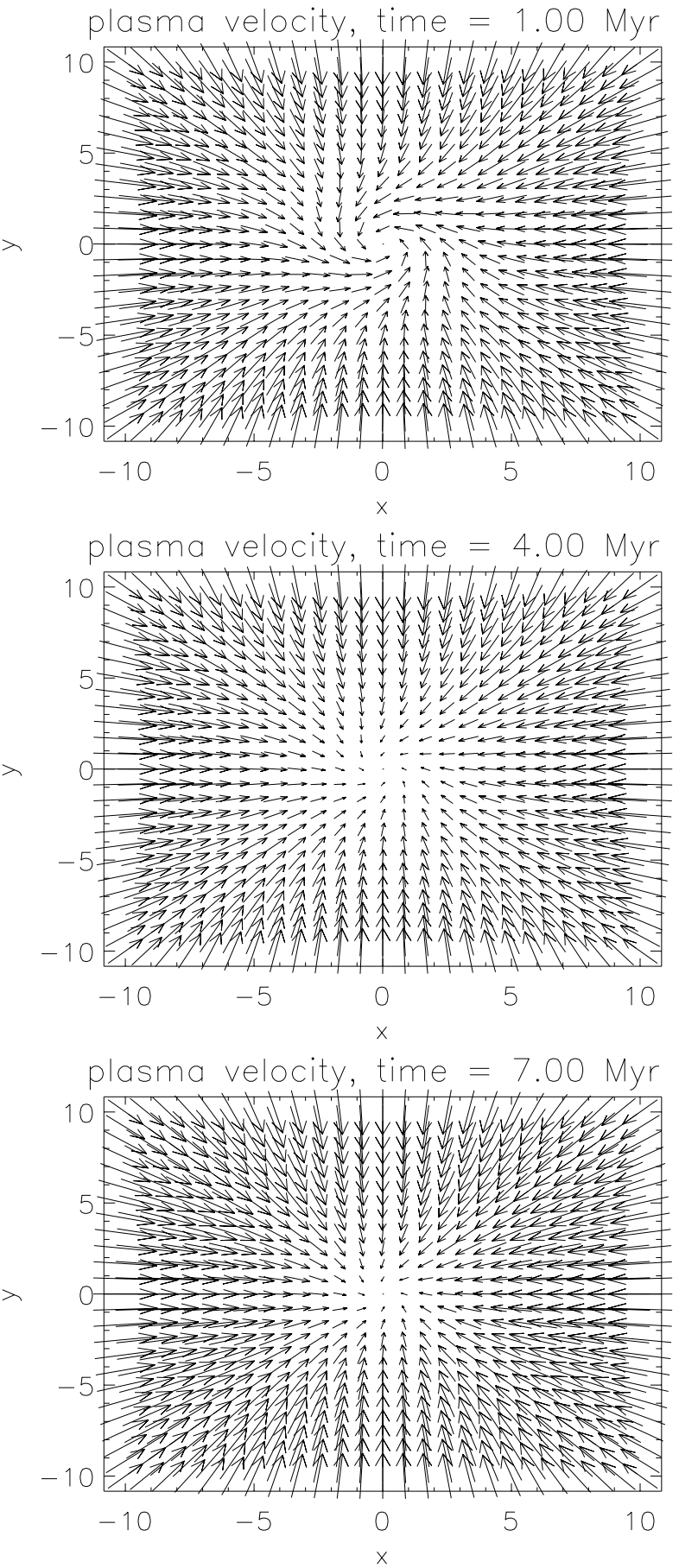

Fig. 5. The temporal evolution of the flow of the ionized fluid component.

and the collisional momentum transfer an ongoing momentum difference and effective field generation is caused as long as the flow is not directed exclusively radially. Other quantities follow in a generic way. One may note, e.g., in our normalization the elementary charge as well as the speed of light that enter in the self-magnetization term in the induction equation $\hat{v}_{\text {en }}$ are not normalized to unity. On the other hand, the gravitational potential is normalized to one. The results that are shown in the next section are gained from simulations in a numerical box of $x \in[-15,15]$ and $y \in[-15,15]$ in units normalized

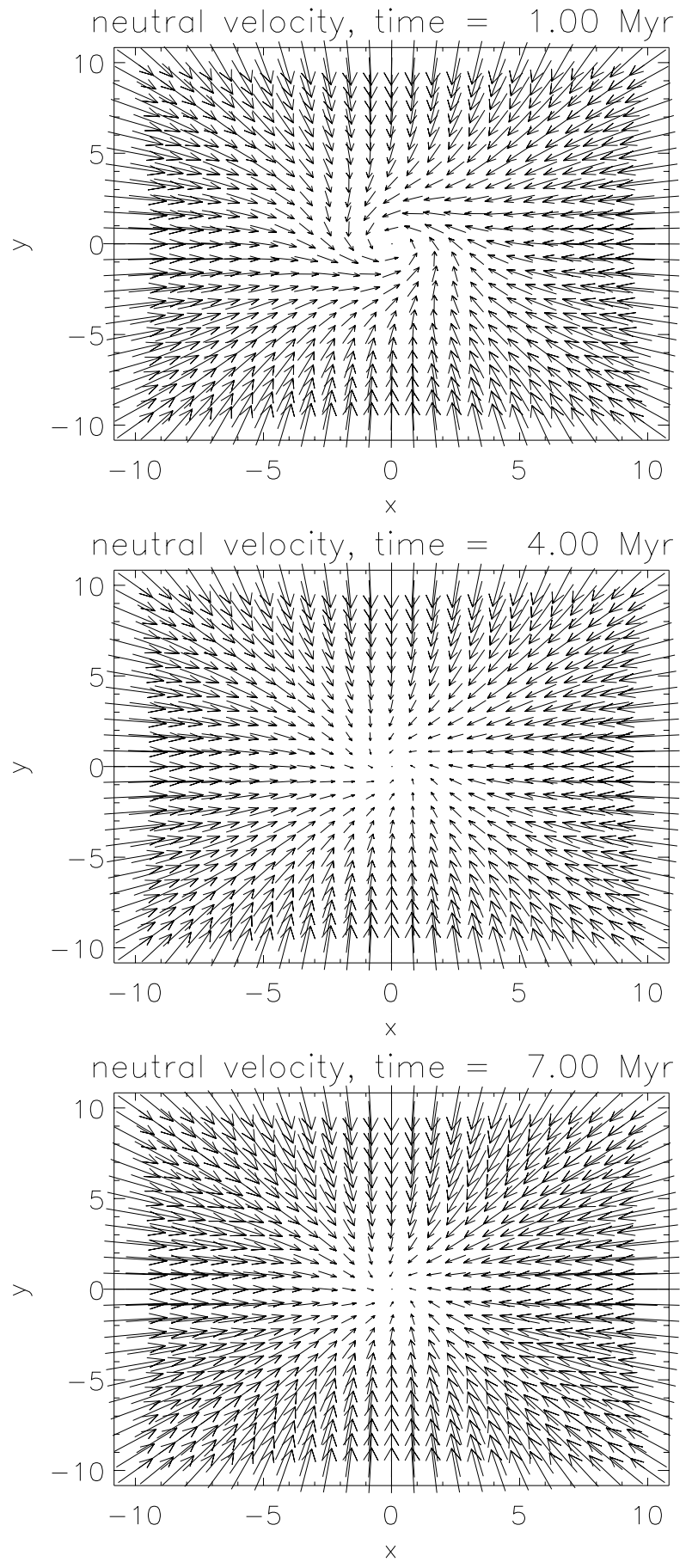

Fig. 6. The temporal evolution of the flow of the neutral fluid component.

to $25 \mathrm{pc}$. An equidistant numerical grid was chosen with a resolution of $221 \times 221$ grid points. The numerical resolution has no significant influence on both the qualitative and quantitative results for reasonable choices. For the boundaries symmetric conditions are prescribed for the mass densities, velocities and magnetic field components. A constant background resistivity according to a magnetic Reynolds number of $S=10^{4}$ was chosen. This choice lies above the small numerical diffusion. The results, in fact, do not differ significantly from the $S=0$ case. 

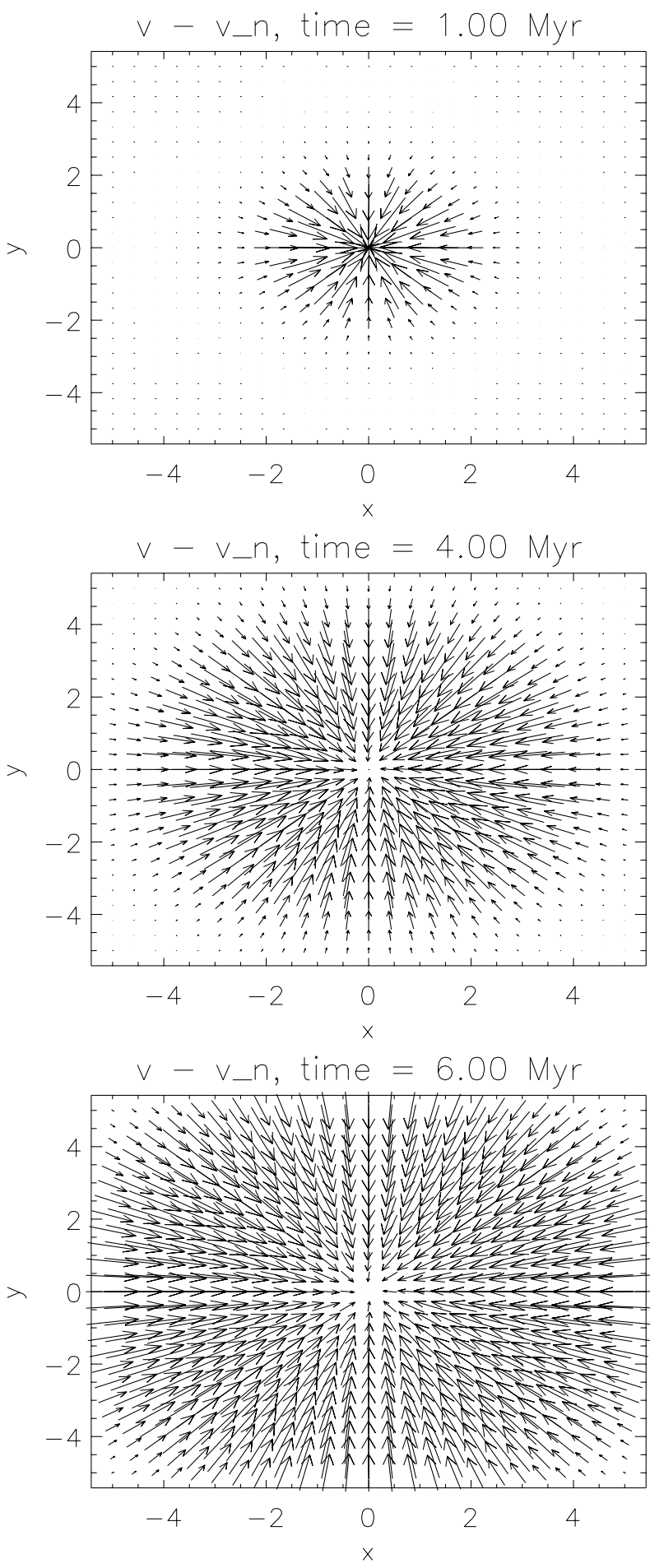

Fig. 7. The temporal evolution of the relative flows of the both fluid components at $t=1 \mathrm{Myr}$ (upper plot), $t=4 \mathrm{My}$ (middle plot) and $t=6 \mathrm{Myr}$ (lower plot).

The same statement holds for the choice of extrapolated boundary conditions instead of symmetric ones.

\section{Generation of magnetic fields}

Our simulations model the dynamics of a rotating selfgravitating partially ionized protogalactic clump that is regarded to be representative for the brick stones of highredshift damped Ly $\alpha$ systems. The collapse dynamics is followed over seven million years which is the same time period as studied in Paper I. First we concentrate on adiabatic dynamics of an ideal gas, i.e. we choose $\gamma=\gamma_{\mathrm{n}}=5 / 3$. The gravitational potential after $1 \mathrm{Myr}$ is displayed in Fig. 2. The shape does not significantly change in time but the amplitude and therefore the gravitational force increases due to the accumulation of matter in the centre of the modelled cloud. The mass densities for the plasma and neutral gas after 4 and $7 \mathrm{Myr}$ are displayed in Figs. 3 and 4. The temporal evolution of the flow patterns of the ionized and neutral components are shown in Figs. 5 and 6, respectively. After 1 Myr (upper plots) the flow patterns show the superposition of the vortical motion and the central inflow caused by the gravitational attractive force. During the dynamical evolution the self-gravitation dominates the flow more and more (middle and lower plots). Different from the earlier heuristic approach in Paper I that did not account for the gravitational pull finite relative shear flows are the combined result of rotation and gravitational acceleration. Significant relative shear flows prevail during the entire shown time period (Fig. 7). The curls of the relative flows are direct measures (Fig. 8) for the magnetic field generation rate. The magnetic field generated by the relative shear flow in the partially ionized rotating and self-gravitating cloud is shown in Fig. 9. During the adiabatically dynamics the cloud regions are heated (Fig. 10). For comparison we also performed simulation runs with isothermal dynamics. This formally implies $\gamma=\gamma_{\mathrm{n}}=1$ in Eqs. (6) and (7). The field generation and central mass accumulation is faster than in the adiabatic case. Figure 11 shows the $z$-component of the generated magnetic field for the isothermal simulations. Again the field strength reaches some $10 \mathrm{fG}$.

Eventually, we present the generated magnetic fields for the initial temperatures $T=10, T_{\mathrm{n}}=1$ and $T=20, T_{\mathrm{n}}=0.5$ (Fig. 12) with a normalization temperature of $1000 \mathrm{~K}$ and $500 \mathrm{~K}$, respectively. The magnetization is comparatively efficient to the $T=T_{\mathrm{n}}=1$ case. The multi-peaked structure is much more pronounced.

\section{Discussion}

We addressed the question of the origin of protogalactic seed fields. If this question is settled, one can be satisfied with current dynamo models that explain how the seed fields are amplified to the observed strengths. Kulsrud and co-workers have shown (Kulsrud et al. 1997) that the Biermann battery mechanism can result in magnetic seed fields that can be amplified by magnetohydrodynamic turbulence. In the present contribution we suggest an alternative mechanism that also should be at work and does not saturate quickly.

Our numerical investigations on the magnetic selfgeneration mechanism due to relative shear flows in a partially ionized self-gravitating vortex show that magnetic seed fields of the strength of some $10^{-14} \mathrm{G}$ can arise in protogalactic clumps on the time scale of some $10^{6} \mathrm{yr}$. These simulations corroborate the model introduced in Paper I and by Wiechen et al. (1999) by now self-consistently including the collapse 

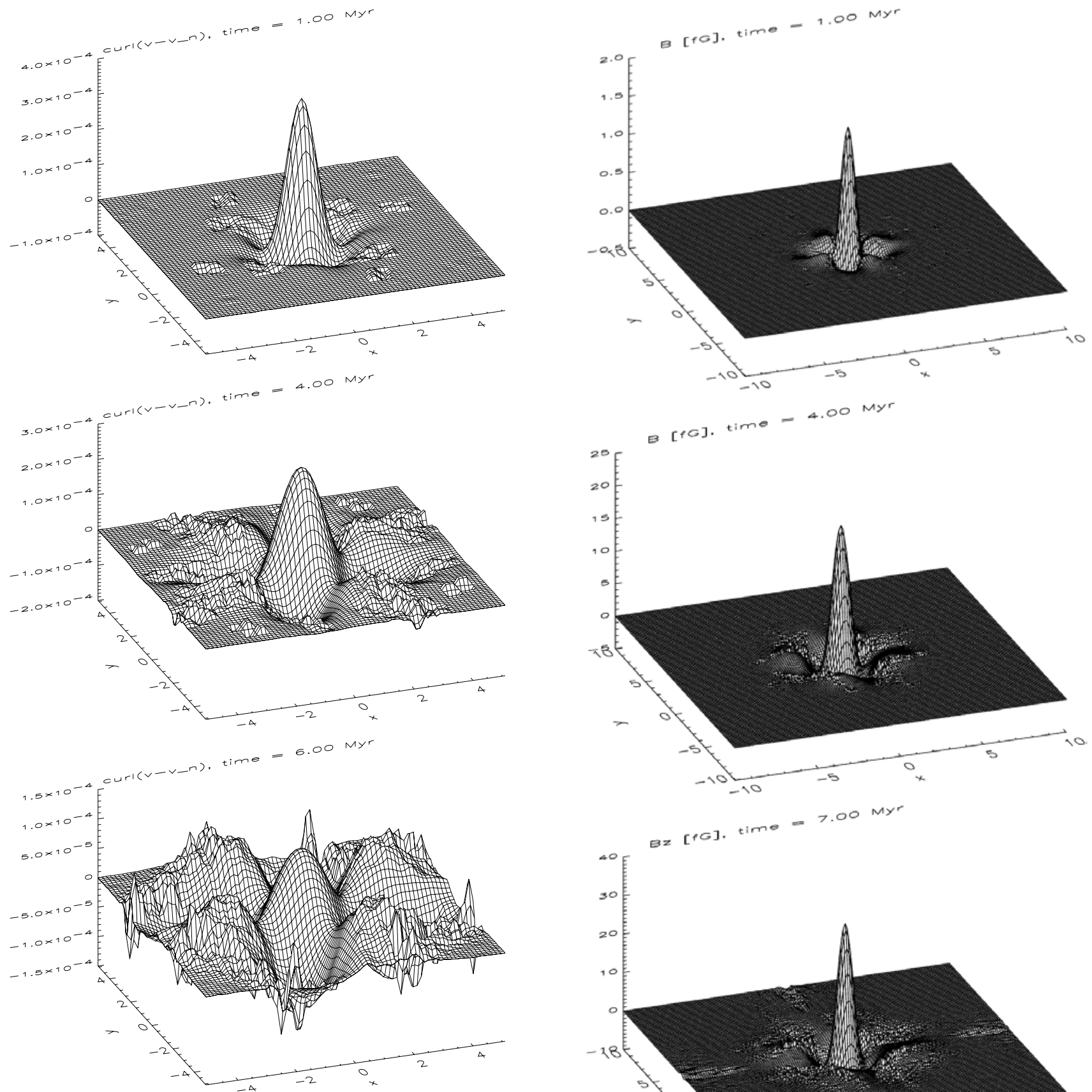

Fig. 8. The temporal evolution of the curl of the relative flows of the both fluid components at $t=1 \mathrm{Myr}$ (upper plot), $t=4 \mathrm{My}$ (middle plot) and $t=6 \mathrm{Myr}$ (lower plot).

dynamics. Different from the latter work the gravitational acceleration is not assumed to be external and constant but is time-dependent and caused by the evolving mass density profile. The gravitational field is the ultimate energy source for the generation of electric currents and thus, the magnetic fields. Subsequent dynamo action can, in principle, amplify galactic magnetic fields up to some $\mu \mathrm{G}$ at a red-shift of $z \approx$ 2-3 (e.g. Lesch \& Chiba 1995). It is worth mentioning that both isothermal and adiabatic protogalactic dynamics result in magnetic field strengths of some $10 \mathrm{fG}$. If in a collapsing Ly $\alpha$ system all clumps are subject to an additional external gravitational potential caused by dark matter, their velocity

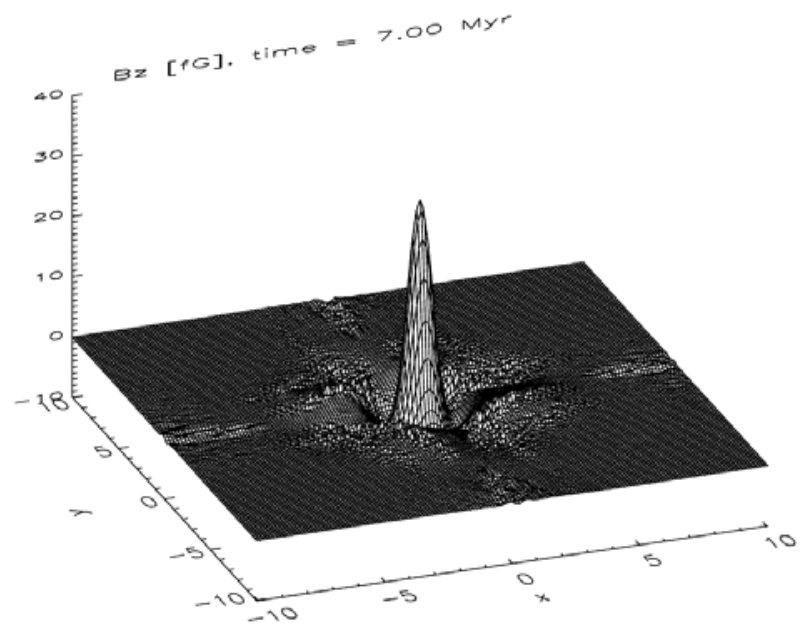

Fig. 9. The temporal evolution of the generated magnetic field. The field strength is measured in units of $10^{-15} \mathrm{G}$. Since the flow is generating shear flow is planar the magnetic field has only one non-vanishing component.

fields should be correlated to some degree. Since the individual clumps can interact via tidal forces in the collapsing region, interclump magnetic fields are expected (Wiechen et al. 1999) in addition to the single magnetization phenomenon discussed in this contribution. Simulations of many self-gravitating rotating clumps in the combined gravitational potential generated by a dark matter halo and the baryonic matter will be a promising topic of future work. 


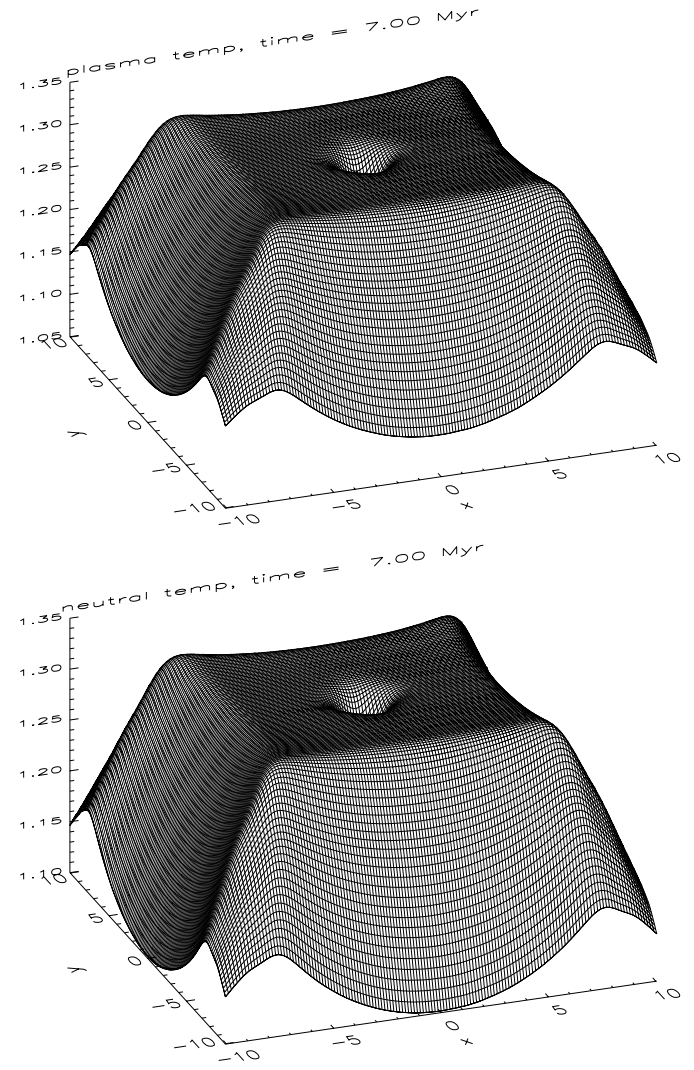

Fig. 10. The temperatures for the ionized (upper plot) and the neutral components (lower plot) of the partially ionized clump after $t=7 \mathrm{Myr}$.

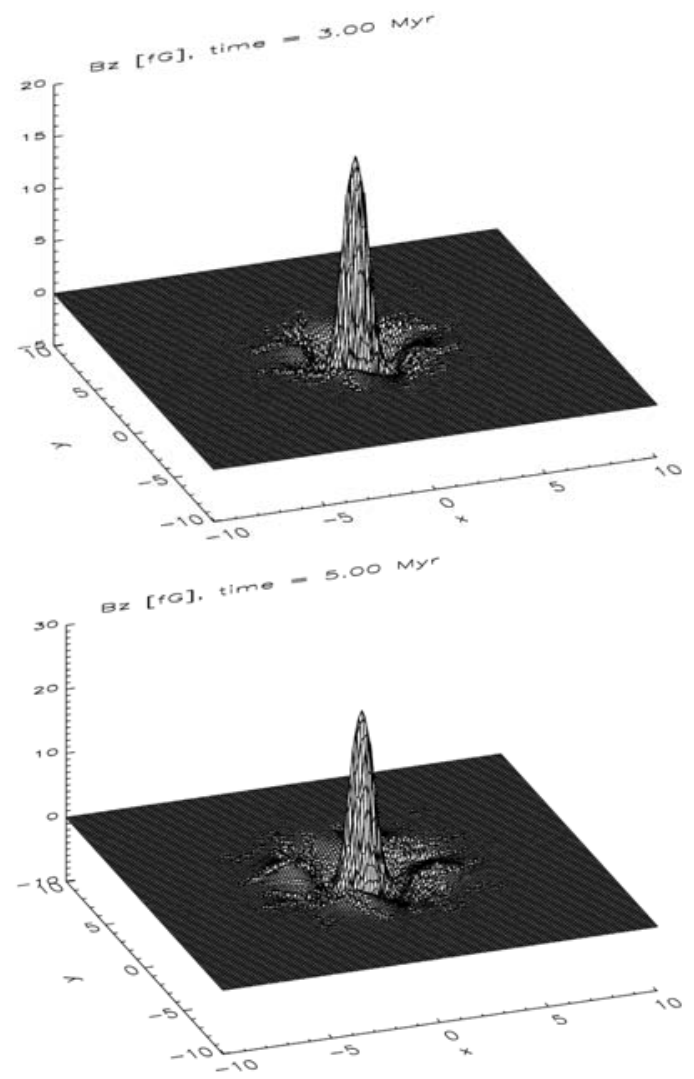

Fig. 11. The temporal evolution of the generated magnetic field for isothermal dynamics. The field strength is measured in units of $10^{-15} \mathrm{G}$.
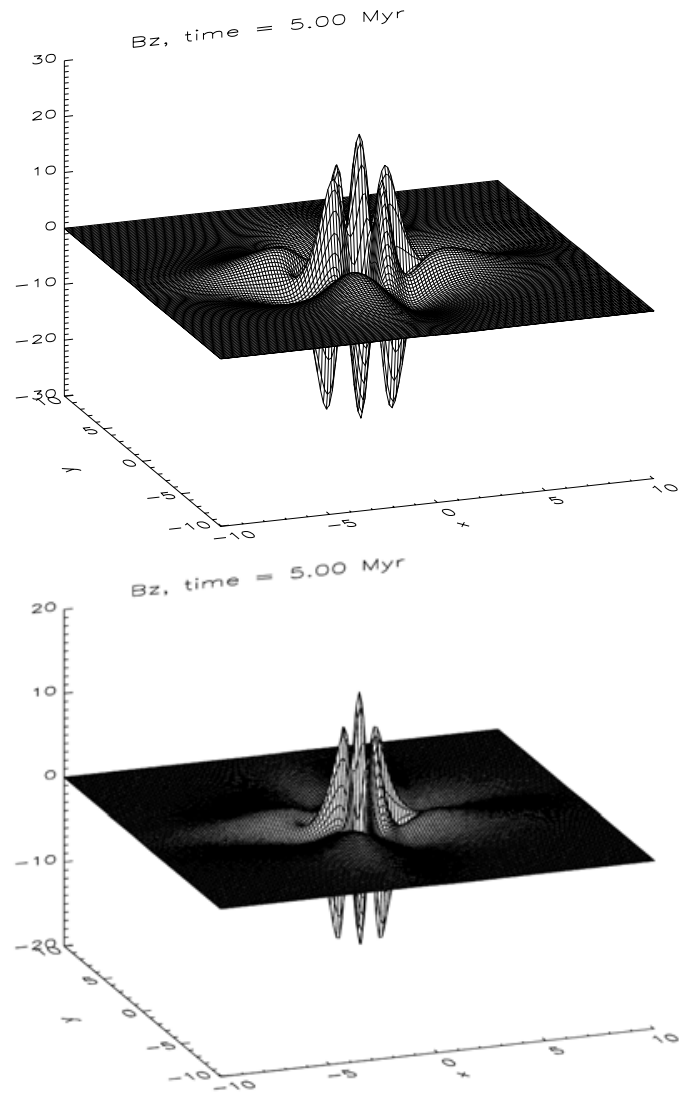

Fig. 12. The generated magnetic field for the initial temperature ratio $T: T_{\mathrm{n}}=10$ (upper plot) and $T: T_{\mathrm{n}}=40$ (lower plot). For the chosen normalizations the neutral gas temperatures are $1000 \mathrm{~K}$ and $250 \mathrm{~K}$, respectively. The field strength is measured in units of $10^{-15} \mathrm{G}$.

\section{References}

Beck, R., Brandenburg, A., Moss, D., Shukurov, A., \& Sokoloff, D. 1996, ARA\&A, 34, 155

Birk, G. T., \& Otto, A. 1996, J. Comp. Phys., 125, 513

Birk, G. T., Kopp, A., \& Shukla, P. K. 1996, Phys. Plasmas, 3, 3564

Goldreich, P., \& Lynden-Bell, D. 1964, MNRAS, 130, 97

Haehnelt, M. G., Steinmetz, M., \& Rauch, M. 1996, ApJ, 465, L95

Huba, J. D., \& Fedder, J. A. 1993, Phys. Fluids B, 5, 3799

Huba, J. D. 1998, NRL Plasma Formulary, p. 39

Kronberg, P. P., Perry, J. J., Zukowski, E. L. H., \& Edwin, L. H. 1992, ApJ, 387, 528

Kronberg, P. P. 1994, Rept. Progr. Phys., 57, 325

Kulsrud, R. M., Cen, R., Ostriker, J. P., \& Ryu, D. 1997, ApJ, 480, 481

Lesch, H., Crusius, A., Schlickeiser, R., \& Wielebinski, R. 1989, A\&A, 217, 99

Lesch, H., \& Chiba, M. 1995, A\&A, 297, 305

Lesch, H., \& Chiba, M. 1997, Fund. Cosm. Phys., 18, 273

Rauch, M., Haehnelt, M. G., \& Steinmetz, M. 1997, ApJ, 481, 601

Rees, M. 1987, QJRAS, 28, 197

Wiechen, H., Birk, G. T., \& Lesch, H. 1998, A\&A, 334, 388

Wiechen, H., Birk, G. T., \& Lesch, H. 1999, Ap\&SS, 264, 347

Wiechen, H., Birk, G. T., \& Lesch, H. 2000, Phys. Plasmas, 7, 701

Wolfe, A. M., Lanzetta, K. M., \& Oren, A. L. 1992, ApJ, 388, 17 